\title{
Frecuencia de cromosoma Filadelfia en niños con leucemia linfoblástica aguda
}

\section{Philadelphia chromosome frequency in children with acute lymphoblastic leukemia}

\author{
Melissa Hernández-Alcaraz, ${ }^{*,+}$ Jesús Ernesto Dueñas-Arias ${ }^{\ddagger}$ \\ * Médico pasante de Servicio Social, Universidad Autónoma de Durango, Campus Mazatlán; \\ ‡ Jefe del Servicio de Genética, Hospital Pediátrico de Sinaloa, México.
}

\begin{abstract}
RESUMEN
Introducción: El oncogén de fusión BCR-ABL, producto del cromosoma Filadelfia $t(9 ; 22)$, es relativamente raro en niños con leucemia linfoblástica aguda (LLA). Su presencia es indicativa de mal pronóstico. Objetivo: Determinar la frecuencia del cromosoma Filadelfia en niños con LLA en el Hospital Pediátrico de Sinaloa, México. Material y métodos: Se revisaron los registros de los experimentos de RTPCR para BCR-ABL de pacientes con diagnóstico de LLA en un periodo de nueve años: 2008-2017. Resultados: De un total de 138 pacientes con LLA, se identificación tres pacientes positivos al cromosoma Filadelfia (2.17\%). Los tres pacientes fueron masculinos, entre dos y ocho años de edad, y catalogados con LLA de alto riesgo. En la actualidad, los tres se encuentran vivos y en tratamiento, aunque han presentado recaídas. Conclusiones: La frecuencia de $2.17 \%$ de cromosoma Filadelfia en niños con LLA que encontramos en este estudio, es muy similar a las descritas en otros países.
\end{abstract}

Palabras clave: Leucemia linfoblástica, cromosoma Filadelfia, pediatría.

\begin{abstract}
Introduction: The BCR-ABL fusion oncogene, a product of the Philadelphia chromosome $t(9 ; 22)$, is relatively rare in children with acute lymphoblastic leukemia (ALL). Its presence is indicative of a poor prognosis. Objective: To determine the frequency of Philadelphia chromosome in children with ALL at the Pediatric Hospital of Sinaloa, Mexico. Material and methods: Nine-year records (2008-2017) of RT-PCR experiments for BCR-ABL of patients diagnosed with $A L L$ were reviewed. Results: Of 138 patients with $A L L$, three Philadelphia chromosome positive patients (2.17\%) were identified. These three patients were male, aged two to eight years, and were classified as having high-risk ALL. Although they have presented relapses, currently all three are alive and remain in treatment. Conclusion: The frequency of $2.17 \%$ of the Philadelphia chromosome in children with ALL that we found in this study, is similar to the figures described in other countries.
\end{abstract}

Keywords: Lymphoblastic leukemia, Philadelphia chromosome, pediatrics.

\section{WWW.medig}

\footnotetext{
+Correspondencia: MHA, mel-hernandez@outlook.com Conflicto de intereses: Los autores declaran que no tienen. Citar como: Hernández-Alcaraz M, Dueñas-Arias JE. Frecuencia de cromosoma Filadelfia en niños con leucemia linfoblástica aguda. Rev Mex Pediatr. 2020; 87(5); 170-175. https://dx.doi. org/10.35366/97170
}

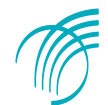

\section{INTRODUCCIÓN}

Las leucemias agudas pediátricas son el tipo de cáncer mejor estudiado desde el punto de vista genético. La aplicación de tecnologías genéticas en la investigación de la leucemia linfoblástica aguda (LLA) tiene una gran importancia pronóstica en la estratificación de riesgo y, por consiguiente, para la elección del tratamiento. ${ }^{1,2}$ 
Los estudios citogenéticos de neoplasias humanas comenzaron en 1960 con el descubrimiento del cromosoma Filadelfia por Nowell y Hungerford, en pacientes con leucemia mieloide crónica (LMC). ${ }^{3} \mathrm{El}$ cromosoma Filadelfia es producto de la translocación recíproca entre el brazo largo del cromosoma 9(q34), donde se localiza el oncogén ABL1, y el brazo largo del cromosoma 22(q11), donde se encuentra el gen BCR, llevando a la formación de la proteína quimérica BCR$\mathrm{ABL}$ y, como resultado de esta fusión, se produce la tirosina cinasa BRC-ABL activa. ${ }^{3}$ Esta alteración es relativamente rara en niños con LLA, pero es un factor de mal pronóstico.

Piu realizó una revisión sistemática donde encontró que la frecuencia de LLA del cromosoma Filadelfia positivo $(\mathrm{Ph}+)$ oscilaba de $1.1 \%$ a $4.0 \% .{ }^{4}$ La frecuencia en Taiwán es de $2.86 \%{ }^{5}$ y en Egipto de $2.4 \%{ }^{6}$ En el caso de México, en los resultados publicados hay diferencias. Verduzco reportó una frecuencia de 19\% de LLA Ph+ en Veracruz, ${ }^{7}$ y Bekker de $1.8 \%$ en la Ciudad de México, ${ }^{8}$ mientras que, en Chiapas, Lepe reportó su ausencia en población predominantemente indígena. ${ }^{9}$

Aun cuando se ha demostrado ampliamente que la detección de BCR-ABL debería ser realizada en todos los niños con LLA al momento del diagnóstico, los estudios moleculares sólo están disponibles en algunos hospitales de tercer nivel. ${ }^{1}$

El propósito de este estudio fue determinar la frecuencia del cromosoma Filadelfia en los pacientes diagnosticados con LLA en el Hospital Pediátrico de Sinaloa (HPS), el cual es un centro de referencia estatal en el noroeste de la República Mexicana.

\section{MATERIAL Y MÉTODOS}

Se realizó un estudio descriptivo, observacional, transversal y retrospectivo en el HPS, el cual incluyó a todos los pacientes diagnosticados con LLA menores de 18 años al momento del diagnóstico y en quienes se realizaron pruebas de diagnóstico molecular para identificar la presencia del gen BCR-ABL1.

Para obtener la información se revisaron los registros del laboratorio de Genética de pacientes con el diagnóstico de LLA en quienes se realizó la detección BCR-ABL mediante reacción en cadena de la polimerasa en tiempo real (RT-PCR), entre el 1 de enero de 2008 y el 31 de diciembre de 2017. Se registró también la edad y sexo de los pacientes.

Para evitar sesgos de selección, se hizo una doble verificación de los pacientes registrados con diagnóstico de LLA, comparándose con el diagnóstico por inmunofenotipo.

\section{Expresión de BCR-ABL1}

En todos los pacientes con LLA se procesaron de manera indistinta muestras de aspirado de médula ósea o sangre periférica. Se recolectaron con ácido

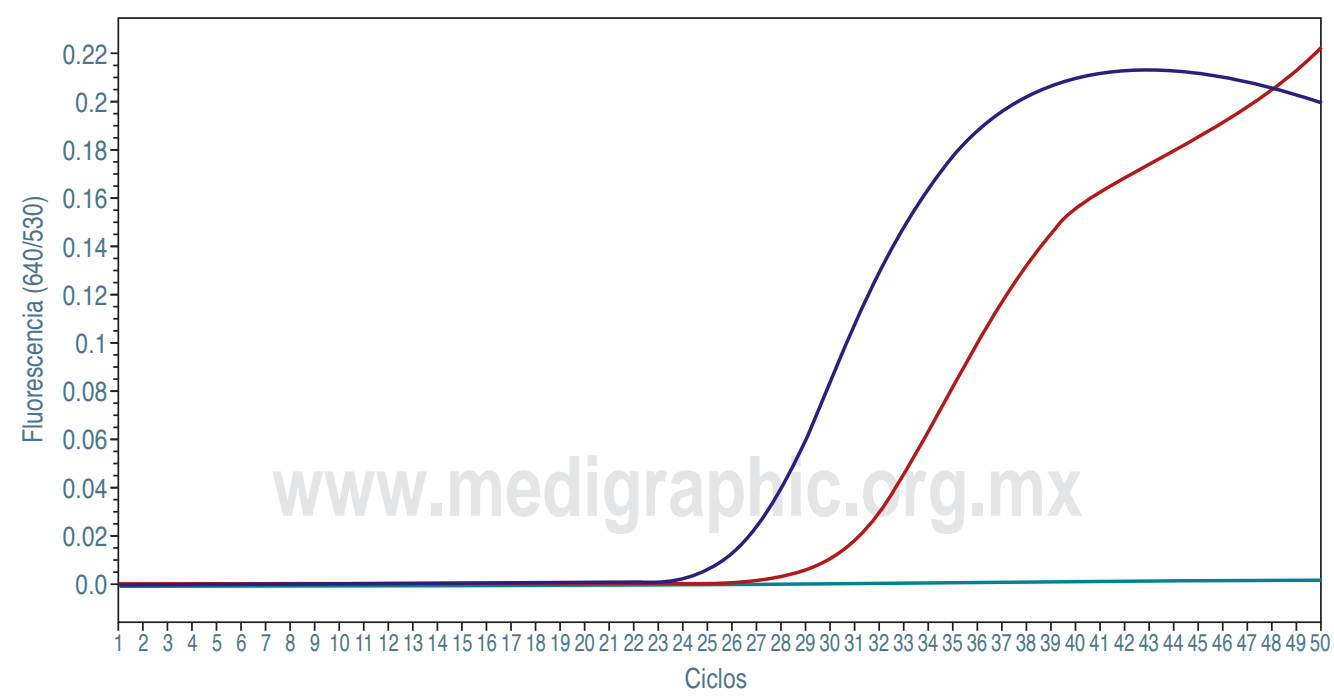

Figura 1: Curva de amplificación PCR-RT para BCR-ABL1 del caso 2. En azul está el estándar (plásmido); en verde, el caso 2; y en rojo, el control negativo. 


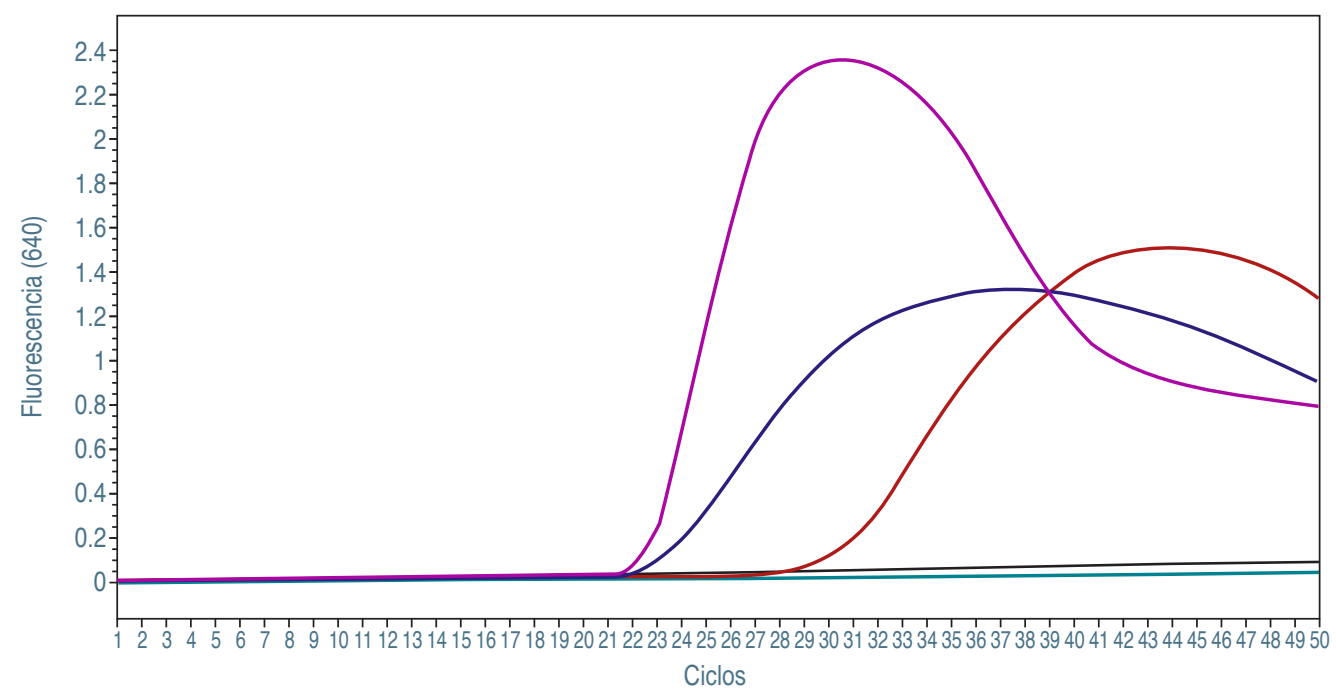

Figura 2: Curva de amplificación para cuantificación de ABL1 y discriminación de transcrito del caso 2. En azul está el control negativo; en verde brillante, rojo y negro el transcrito mayor, menor y micro, respectivamente; en rosa estándar está el plásmido; en verde oscuro el gen de referencia (ABL1).

etilendiaminotetraacético (EDTA), se obtuvo ARN con el kit de extracción y, a partir de éste, se sintetizó ADN complementario (cDNA) con kits de la marca ROCHE $^{\circledR}$. La RT-PCR se llevó a cabo mediante el kit LightMix ${ }^{\circledR}$ para BCR-ABL t(9;22). Se realizó la mezcla para PCR, utilizando un LightCycler ${ }^{\circledR}$, donde se agregó $1 \mu \mathrm{L}$ de cada primer, cumpliéndose las condiciones siguientes: desnaturalización inicial a $95{ }^{\circ} \mathrm{C}$ durante 10 minutos, 50 ciclos en variaciones de temperatura desde $95{ }^{\circ} \mathrm{C}$ por cinco segundos, 60 ${ }^{\circ} \mathrm{C}$ por 10 segundos, $72{ }^{\circ} \mathrm{C}$ por 20 segundos y una extensión final de $40{ }^{\circ} \mathrm{C}$ por 30 segundos. Los productos amplificados se muestran en tiempo real, donde el estándar (plásmido) ayuda a contabilizar los productos detectados por fluorescencia, así como para determinar el porcentaje de expresión de BCR-ABL1 en caso de ser positivo.

\section{RESULTADOS}

De un registro de 155 análisis moleculares para BCRABL1, 17 fueron excluidos por tratarse de muestras de seguimiento, lo que dejó un total de 138 pacientes con LLA. De este total, 72 eran pacientes masculinos (52.2\%) y 66 femeninos (47.8\%); todos entre siete meses y 17 años, con un promedio de edad de seis años 10 meses. Del total, sólo hubo tres pacientes (2.17\%) positivos para Filadelfia. A continuación, se presenta una breve descripción de cada uno de los tres.

\section{Caso 1}

Paciente masculino de dos años y tres meses, que fue referido de Los Mochis, Sinaloa. Acudió inicialmente por edema palpebral de tres días de evolución. Fue llevado al Hospital General de Los Mochis donde se tomó muestra de laboratorio y fue referido al HPS por sospecha de leucemia aguda. Al momento de su ingreso: hemoglobina $6.4 \mathrm{~g} / \mathrm{dL}$, hematocrito $19 \%$, plaquetas 221,000 y leucocitos 330,540 , siendo el $36 \%$ de blastos.

En marzo de 2015, mediante un aspirado de medula ósea, se estableció diagnóstico de LLA-L1. El inmunofenotipo: CD19, CD20, CD22, CD10, CD34, TdT, HLA-DR, CD13, CD33, MPO positivos, que junto con BCR-ABL1 positivo se clasificó LLA de alto riesgo. ${ }^{10}$

\section{Caso 2}

Paciente de ocho años de edad referido de Los Mochis, Sinaloa. Tuvo un cuadro de 15 días de evolución, caracterizado por palidez, pérdida de peso, coluria, acolia y dolor abdominal; se descartó hepatitis viral. Posteriormente presentó tres episodios de hematemesis, artralgias y fiebre; fue referido al HPS por bicitopenia e hiperleucocitosis. Al momento de su ingreso: hemoglobina 4.1 $\mathrm{g} / \mathrm{dL}$, hematocrito $13 \%$, plaquetas 15,000 y leucocitos 149,950; neutrófilos 0\%, linfocitos $42 \%$ y blastos de $53 \%$. 
En mayo de 2016, con aspirado de medula ósea, se estableció el diagnóstico de LLA-L2. El inmunofenotipo: CD19, CD22, cCD79a, CD10, CD34, TdT positivos, que junto con BCR-ABL1 positivo (Figuras 1 a 3), se clasificó como LLA de alto riesgo. ${ }^{10}$

\section{Caso 3}

Paciente de ocho años y un mes de edad, que fue referido de Navolato, Sinaloa. El cuadro clínico fue con fiebre, hiporexia, dolor ocular, astenia y adinamia. En un principio fue valorado en medio privado y manejado con antipirético y antibiótico. Fue referido al HPS por crisis convulsivas y fiebre. $\mathrm{Al}$ momento de su ingreso: hemoglobina $4.6 \mathrm{~g} / \mathrm{dL}$, hematocrito $18.8 \%$, plaquetas 13,000 y leucocitos 380,550 , segmentados $6 \%$, linfocitos $15 \%$, blastos 76 .

En octubre de 2015, mediante un aspirado de médula ósea, se estableció el diagnóstico de LLA-L1. El inmunofenotipo: CD19, CD20, CD22, cCD79a, CD10, CD34, TdT, HLA-DR, CD36 positivos y BCR-ABL1 positivo, se clasificó como LLA de alto riesgo. ${ }^{10}$

Los tres pacientes se encuentran vivos y en tratamiento, pero todos han presentado recaídas a pesar de haber sido tratados con anticuerpo monoclonal específico para su patología.
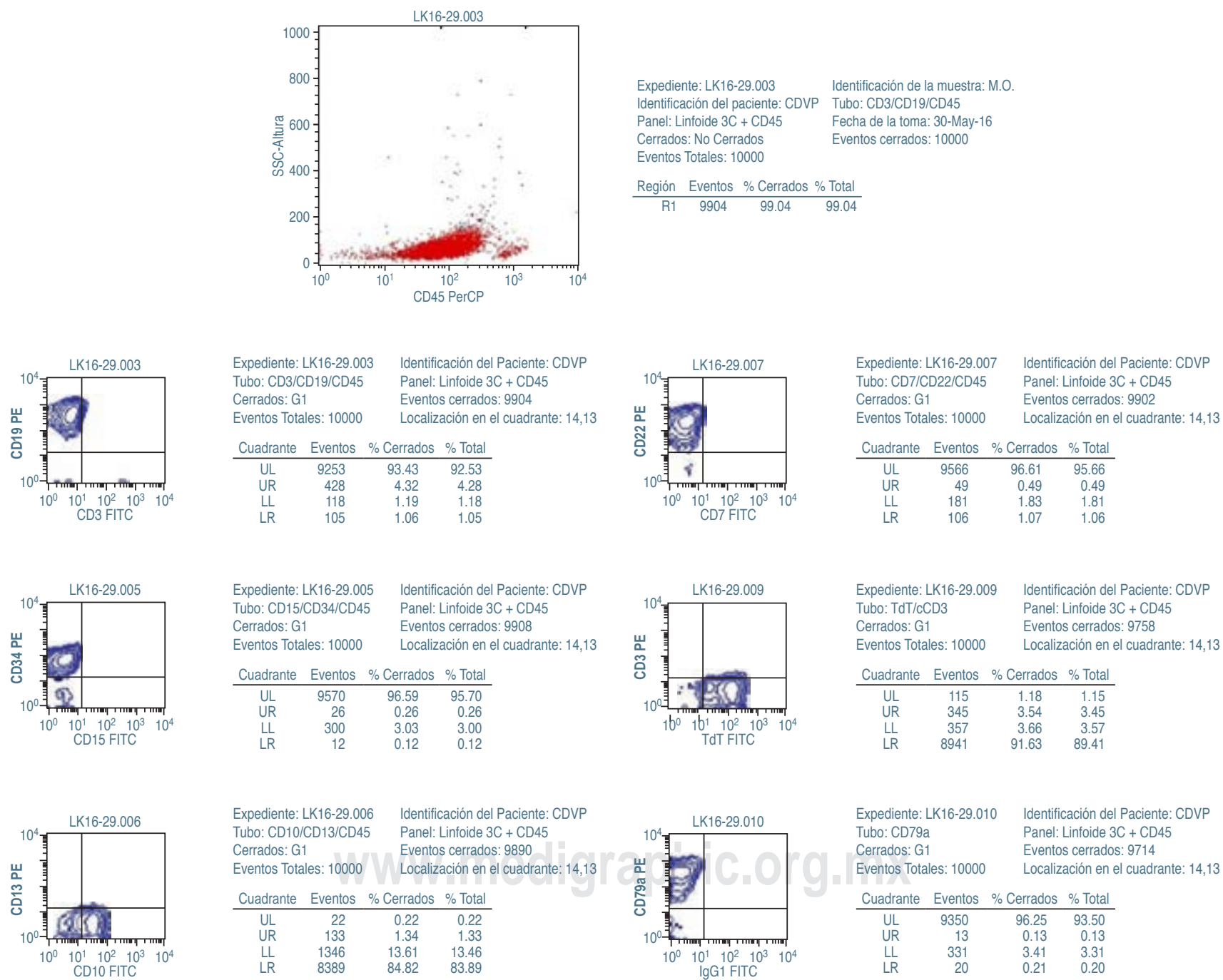

Figura 3: Gráficas obtenidas por citometría de flujo del caso 2, en las que se observa población de blastos tipo B destacadas con CD45 positivo. Las gráficas específicas indican poblaciones positivas de los marcadores CD19, CD22 y CD79a; y los marcadores indicativos de inmadurez celular CD34, CD10 y TdT corresponden a una línea de células inmaduras de tipo B. 


\section{DISCUSIÓN}

Las tasas de cáncer infantil varían entre razas y grupos étnicos ${ }^{6,11} \mathrm{y}$, de acuerdo con ciertos autores, los niños hispanos tienen el peor pronóstico en comparación con niños blancos y asiáticos. ${ }^{12-14}$ Del total, la LLA es de las neoplasias más frecuentes en la infancia.

En el presente estudio, la frecuencia de cromosoma Filadelfia en niños con LLA fue de $2.17 \%$, la cual es similar a lo reportado en Taiwán $(2.86 \%)^{5}$ y Egipto $(2.4 \%){ }^{6}$ asimismo, se encuentra dentro del rango reportado en una revisión sistemática de 1.1-4.0\%. ${ }^{4}$ En México, estudios previos han descrito frecuencias muy amplias que van de 0 hasta $19 \%$. $^{7-9}$

La supervivencia de los pacientes con LLA se ha incrementado notablemente en las últimas décadas, pasando de una supervivencia de menos de $10 \%$ en los años sesenta a presentar con los tratamientos actuales una supervivencia libre de enfermedad superior a $80 \%$ en la mayoría de los casos. ${ }^{15,16}$ Estas tendencias a mejorar la supervivencia no sólo se deben a los protocolos terapéuticos actuales, sino al óptimo manejo diagnóstico del paciente, que van desde la sospecha clínica hasta el empleo de las diferentes metodologías diagnósticas disponibles, tales como citogenética, citometría de flujo y biología molecular, que ayudan a clasificar el riesgo de cada paciente. En este contexto, la presencia del cromosoma Filadelfia confiere mal pronóstico, de ahí que se clasifican con LLA de alto riesgo.

Si bien, no hay una recomendación de una terapia específica en el tratamiento de pacientes con LLA de alto riesgo, distintos estudios buscan encontrar coadyuvantes al régimen de quimioterapia que ayuden a disminuir el riesgo de recaída y aumentar la supervivencia, mientras se disminuyen los efectos adversos y complicaciones inherentes a estos agresivos tratamientos. ${ }^{17-19}$

Por último, consideramos pertinente comentar que, a pesar de que la frecuencia del cromosoma Filadelfia es baja, para todos los pacientes con LLA debería estar disponible su detección para establecer con mayor precisión el riesgo de cada paciente, con la finalidad de otorgar terapias específicas y, en lo posible, mejorar su pronóstico.

\section{REFERENCIAS}

1. Jiménez-Morales S, Hidalgo-Miranda A, Ramírez-Bello J. Leucemia linfoblástica aguda infantil: una aproximación genómica. Bol Med Hosp Infant Mex. 2017; 74(1): 13-26.
2. Jha S, Kumar D, Kaul JM, Singh T, Dubey AP. Cytogenetic pattern profiling in cases of Acute Lymphoblastic Leukemia in pediatric age group. J Anat Soc India. 2017; 66(1): 48-53.

3. Gallegos-Arreola MP, Borjas-Gutiérrez C, Zúñiga-González GM, Figuera LE, Puebla-Pérez AM, García-González JR. Pathophysiology of acute lymphoblastic leukemia. In: MejíaAranguré JM. Clinical epidemiology of acute lymphoblastic leukemia-from the molecules to the clinic. Rijeka, Croatia: InTechOpen; 2013, pp. 48-50.

4. Pui C-H, Carroll WL, Meshinchi S, Arceci RJ. Biology, risk stratification, and therapy of pediatric acute leukemias: an update. J Clin Oncol. 2011; 29(5): 551-565.

5. Liang DC, Yang CP, Lin DT, Hung IJ, Lin KH, Chen JS et al. Longterm results of Taiwan Pediatric Oncology Group studies 1997 and 2002 for childhood acute lymphoblastic leukemia. Leukemia. 2010; 24(2): 397-405.

6. Mikhail FM, Serry KA, Hatem N, Mourad ZI, Farawela HM, El Kaffash DM et al. AML1 gene over-expression in childhood acute lymphoblastic leukemia. Leukemia. 2002; 16 (4): 658-568.

7. Verduzco-Rodríguez L, Verduzco-Aguirre HC, López-Ariza B. Leucemia linfoblástica aguda hiperdiploide en niños. Revista de Hematología. 2012; 13: 172-176.

8. Bekker-Méndez VC, Miranda-Peralta E, Núñez-Enríquez JC, Olarte-Carrillo I, Guerra-Castillo FX, Pompa-Mera EN et al. Prevalence of gene rearrangements in Mexican children with acute lymphoblastic leukemia: a population study-report from the Mexican Interinstitutional Group for the identification of the causes of childhood leukemia. Biomed Res Int. 2014; 2014: 210560.

9. Lepe-Zúñiga JL, Jerónimo-López FJ, Hernández-Orantes JG. Características citopatológicas de la leucemia aguda en el Hospital de Especialidades Pediátricas de Chiapas, México. Bol Med Hosp Infant Mex. 2017; 74(2): 122-133.

10. Schultz KR, Pullen DJ, Sather HN, Shuster JJ, Devidas M, Borowitz MJ et al. Risk- and response-based classification of childhood B-precursor acute lymphoblastic leukemia: a combined analysis of prognostic markers from the Pediatric Oncology Group (POG) and Children's Cancer Group (CCG). Blood. 2007; 109(3): 926-935.

11. Chow EJ, Puumala SE, Mueller BA, Carozza SE, Fox EE, Horel $S$ et al. Childhood cancer in relation to parental race and ethnicity: a 5-state pooled analysis. Cancer. 2010; 116(12): 3045-3053.

12. Bhatia S, Sather HN, Heerema NA, Trigg ME, Gaynon PS, Robison LL. Racial and ethnic differences in survival of children with acute lymphoblastic leukemia. Blood. 2002; 100(6): 1957-1964.

13. Aldrich MC, Zhang L, Wiemels JL, Ma X, Loh ML, Metayer C et al. Cytogenetics of Hispanic and White children with acute lymphoblastic leukemia in California. Cancer Epidemiol Biomarkers Prev. 2006; 15(3): 578-581.

14. Lim JYS, Bhatia S, Robison LL, Yang JJ. Genomics of racial and ethnic disparities in childhood acute lymphoblastic leukemia. Cancer. 2014; 120(7): 955-962.

15. Hunger SP, Lu X, Devidas M, Camitta BM, Gaynon PS, Winick NJ et al. Improved survival for children and adolescents with acute lymphoblastic leukemia between 1990 and 2005: a report from the children's oncology group. J Clin Oncol. 2012; 30(14): 1663-1669.

16. Rabin KR, Poplack DG. Management strategies in acute lymphoblastic leukemia. Oncology. 2011; 25(4): 328-335.

17. Biondi A, Schrappe M, De Lorenzo P, Castor A, Lucchini G, Gandemer $V$ et al. Imatinib after induction for treatment of children and adolescents with Philadelphia-chromosomepositive acute lymphoblastic leukaemia (EsPhALL): a 
randomised, open-label, intergroup study. Lancet Oncol. 2012; 13(9): 936-945.

18. Shen S, Chen X, Cai J, Yu J, Gao J, Hu S et al. Effect of dasatinib vs imatinib in the treatment of pediatric philadelphia chromosomepositive acute lymphoblastic leukemia: a randomized clinical trial. JAMA Oncol. 2020; 6(3): 358-366.
19. Biondi A, Gandemer V, De Lorenzo P, Cario G, Campbell M, Castor A et al. Imatinib treatment of paediatric Philadelphia chromosome-positive acute lymphoblastic leukaemia (EsPhALL2010): a prospective, intergroup, open-label, single-arm clinical trial. Lancet Haematol. 2018; 5(12): e641652. 